\title{
High Energy Accelerators in Medicine
}

\author{
F.J.M. Farley and P. Mandrillon \\ Laboratoire du Cyclotron, Centre Antoine Lacassagne \\ Nice, France
}

Treating tumours with charged particles ranging from protons to light ions has many advantages, but has been little used because the dedicated accelerator facilities now planned or under construction are lacking.

Improving the quality of medical treatment by means of new irradiation technologies is one of the radiotherapist's constant concerns. Each improvement in techniques to produce radiation and to control doses has led to an increase in the number of patients cured and to a reduction of adverse reactions. The replacement of $200 \mathrm{keV} x$-rays by $\gamma$-rays from ${ }^{60} \mathrm{Co}$, which were in turn superseded by $x$-rays from electron linacs, illustrate the progress. The clinical benefits are essentially due to an improved dose distribution of the radiation giving a lower dose to normal tissues for the same dose to a tumour. The latest techniques have made radiotherapy the most widely used form of cancer treatment, given to two out of every three patients. Approximately 25 percent of cured cancer patients are still alive after five years thanks to radiotherapy alone, and a further 12 percent are cured by this form of treatment combined with other methods (a five-year survival is currently accepted as "proof of cure").

It was Robert Wilson, a student of Ernest Lawrence, who realized in 1946 at Harvard University in the USA that protons travel in almost straight lines and stop at a definite depth (they have the same "range"), and that this could lead to a new radiotherapeutic tool. He made radiotherapy one of the principle tasks of

Francis J.M. Farley is a Consultant at the Laboratoire du Cyclotron, Centre Antoine Lacassagne, 227 avenue de la Lanterne, F-06200 Nice, one of 20 centres for cancer treatment in France. He studied at Cambridge University in the UK, receiving his Ph.D. in physics in 1949 after wartime research on radar and a year at the Chalk River Labs in Canada. He was successively Senior Lecturer, Auckland University, New Zealand, a member of CERN staff and Dean of the Royal Military College, Shrivenham, UK, and has held several temporary appointments since 1982 .

Pierre Mandrillion heads the Laboratoire du Cyclotron at the Centre Antoine Lacassagne, Nice, where he was responsible for building the MEDICYC cyclotron, dedicated to proton and neutron therapy, and was Project Leader of the EULIMA initiative. He received his Ph.D. from Grenoble University in 1971 and then helped upgrade CERN's synchrocyclotron.

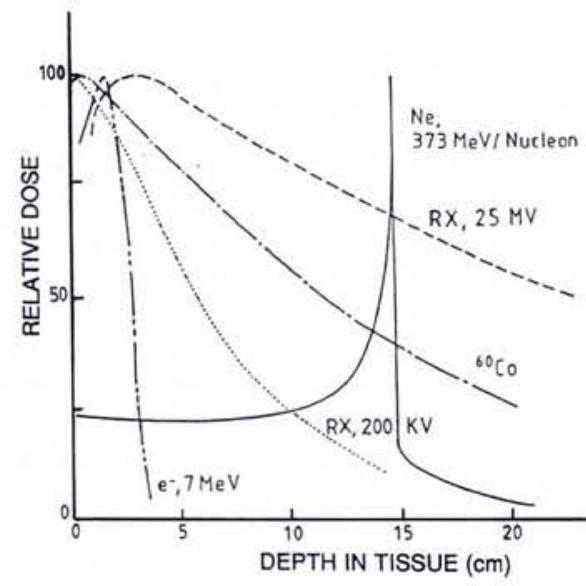

Fig. 1 - Depth-dose distribution for different radiations in Gy normalized to 100 at the peak ( $R X$ is $X$-rays).

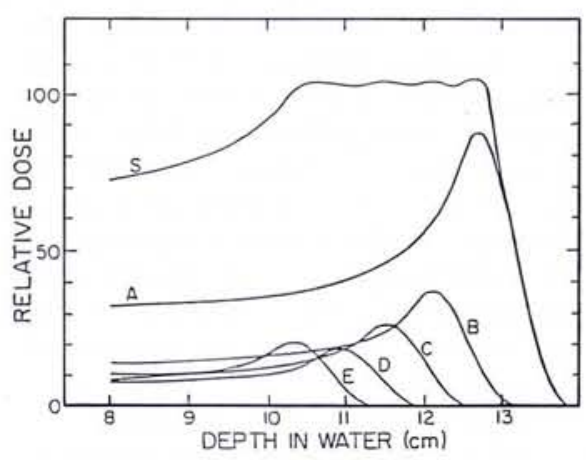

Fig. 2 - Overlapping Bragg peaks for protons. A-E for mono-energetic protons of decreasing energies, which combine to give the total dose distribution S.

the Harvard cyclotron, which since 1961 has treated more patients than any other accelerator facility.

Following the successful pioneering work carried out with accelerators built for physics research, machines dedicated to this new radiotherapy are planned or are already under construction. Some 45 years after Wilson's pioneering work, the first hospital-based, dedicated, proton accelerator is currently being commissioned at the Loma Linda University Medical Center, USA, to treat 1000 patients each year.

We shall discuss the rationale for radiotherapy based on light ions, which essen- tially exploits two factors (improved physical selectivity and improved biological effectiveness [1]), and the high energy accelerators and the beam delivery systems that are needed.

\section{Physical Selectivity}

The treatment of cancer with radiation is at present confined almost entirely to $\mathrm{x}$-rays, $\gamma$-rays and electrons. However, these three types of radiation do not provide ideal dose distributions for treating tumours located deep inside the body. Fig. 1 shows the dose distribution achieved in practice with electrons, soft x-rays, ${ }^{60} \mathrm{Co} \gamma$-rays and megavolt $\mathrm{x}$-rays [2]; the maximum dose is in the more superficial tissues and there would be a substantial dose beyond the tumour. Some unwanted radiation (not shown in the diagram) also passes outside the main beam.

The momentum transferred (force $x$ time) to a quasi-stationary electron in the medium as an ion of charge $Z$ and velocity $v$ passes is proportional to $Z / v$. The energy transferred is therefore proportional to $(Z / V)^{2}$. The ionization density (called LET, the linear energy transfer) rises as the ion slows down, until at the very end of the range the ionic charge is reduced by electron pick-up and the ionization falls rapidly to zero. This gives rise to the Bragg curve of ionization, seen in Fig. 1 for $\mathrm{Ne}$ ions, where the maximum dose is delivered in a narrow peak at the end of the range at a position that can be precisely controlled.

The dependence of the depth of penetration on $Z^{2}$ is also an important feature. It implies that for equal velocities the ionization density for oxygen ions $(Z=8$, atomic number $A=16$ ) is 64 times greater than for protons. But as the kinetic energy is only 16 times larger (for the same velocity) the range will be four times smaller. So the Bragg peak for ions is more narrowly concentrated at the end of the range than for protons. On the other hand, much higher energies are needed to achieve the range in tissue required to reach deepseated tumours.

The Bragg peak is only a few millimetres wide and must be broadened in order to give a uniform dose to a tumour several centimetres thick. Fig. 2 indicates sche- 


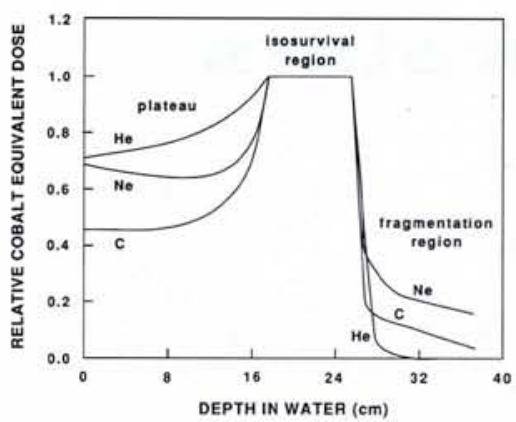

Fig. 3 - Extended Bragg peaks for helium, carbon and neon beams adjusted to give the same dose at distances of 17 to $25 \mathrm{~cm}$ in depth.

matically how by superposing beams of different energies [3] the peak can be enlarged in modulating the beam energy. In practice, the beam energy is most easily modulated by passing the beam through a variable absorber, for example, a disc of varying thickness which is rotated in the beam.

Typical Bragg peaks extended in this way, for various particles are given in Fig. 3 , taking into account the biological effects discussed below [4]. One should note that the dose in the "plateau" region in front of the extended Bragg peak depends on the projectile, carbon being apparently the best in that its plateau lies at the lowest dose. At longer ranges beyond the peak the effect of projectile fragmentation can be seen. Some of the incoming ions break up as they pass through the target, giving fragments of roughly the same velocity. As these fragments usually carry a smaller electric charge, they penetrate further than the original projectile. Helium shows very little fragmentation, which becomes increasingly more severe on moving up the periodic table.

Another advantage of the light ions is that they travel in almost straight lines so very sharp collimation is possible. The lateral deflection due to multiple Coulomb scattering is reduced as the mass of the particle increases [5]. For carbon at a depth of $20 \mathrm{~cm}$, the sideways scattering is only $1.5 \mathrm{~mm}$, compared to $6.5 \mathrm{~mm}$ for protons. Electrons, on the other hand, are scattered so much that they are useful only for treating tumors located relatively close to the surface.

The inconvenience of the light ions is that much higher energies are required to obtain the $20 \mathrm{~cm}$ range needed to reach

Table 1 - Ion energies for a range of $20 \mathrm{~cm}$ in water

\begin{tabular}{|cccc|}
\hline & $\begin{array}{c}\text { Energy } \\
\text { MeV/nucleon }\end{array}$ & $\begin{array}{c}\text { Magnetic } \\
\text { rigidity } \\
\text { Tm }\end{array}$ & $\begin{array}{c}\text { Intensity } \\
\text { required } \\
\text { pps }\end{array}$ \\
\hline $\mathbf{p}$ & 175 & 2.00 & $2.10^{10}$ \\
$\mathrm{He}$ & 175 & 4.00 & $5.10^{9}$ \\
$\mathbf{C}$ & 330 & 5.67 & $1.10^{9}$ \\
$\mathbf{0}$ & 400 & 6.34 & $7.10^{8}$ \\
$\mathrm{Ne}$ & 470 & 6.98 & $5.10^{8}$ \\
\hline
\end{tabular}

deep-seated tumours. Ranges for different light ions are plotted in Fig. 4 as a function of the kinetic energy per nucleon [6] to show how the required energy increases sharply with the range.

The energies and magnetic rigidities ( $=B R$, where $R$ is the bending radius in a magnetic field $B$, usually expressed in $\mathrm{Tm}$ ) for a range of $20 \mathrm{~cm}$ are given in Table 1 . The magnetic rigidity fixes the bending radius of the particles, and therefore determines the size of the accelerator and the beam delivery system. The number of particles per second (pps) required to deliver a dose of $5 \mathrm{~Gy}$ over a volume of two litres in one minute is given in the final column.

There are, of course, fruitful applications for low energy accelerators in treating tumours which are less deep-seated, when precise dose localization is mandatory. An outstanding example is the use of $65 \mathrm{MeV}$ protons (range $35 \mathrm{~mm}$ ) for melanomas of the eye, pioneered at Harvard University, USA, and now adopted at several other centres. Tumours are kept under control in almost all cases, and the irradiated eyes are conserved; over half the treated patients can see as well or better than before treatment [7].

\section{Biological Effectiveness}

Different cells respond differently to radiation and a loosely defined parameter called "radiosensitivity" gives an indication of the response. The biological effects of light ions have been investigated for many years at Berkeley in the USA. Densely ionizing particles, when they hit a cell, produce more concentrated local damage and have a more immediately lethal effect than the scattered minor lesions inflicted by weakly ionizing particles. As a result, radiation with a high linear energy transfer (LET) exhibits differences in the effects on biological tissue, when compared to lowLET radiation: these may be summarized as follows $[1,8]$ :

- dividing cells are more sensitive;

- some types of tumour cells are essentially insensitive;

- large tumours de-oxygenated in the centre are less sensitive;

- healthy cells can repair themselves if the radiation is divided into fractions administered at intervals.

For high-LET radiation, all cells respond more or less similarly and repair mechanisms are less effective.

The last characteristic has important consequences for oxygen deficient (hypoxic) cells, which are, in general, less sensitive to radiation and are often present at the centres of large, poorly vascularised, tumours (i.e., tumours lacking arteries and vessels to provide a good oxygen supply). The effect of oxygen is less important for high-LET particles, see Fig. 5 [9], so hypoxic cells do not survive highLET irradiation.

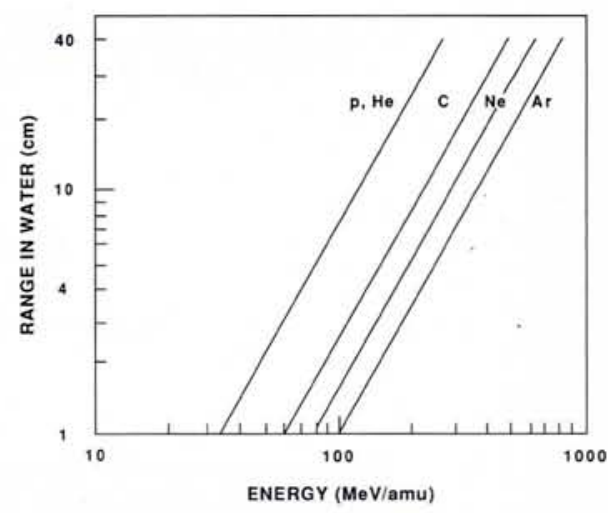

Fig. 4 - Range in water versus kinetic energy per nucleon for various beams.

It follows that high-LET, light ion treatment is particularly useful for radioresistant tumours, insensitive to normal x-rays. These will often be slowly growing tumours with cells which only rarely undergo cell division, or large, de-oxygenated tumours. On the other hand [1], there is smaller difference between the sensitivities of cancerous and normal tissues so the beam must be very carefully delivered in order to impart the minimum possible dose to healthy cells. By the same token, because the amount of repair after irradiation is smaller, fractionation schemes are less helpful in giving healthy tissue a chance to recover.

In summary, the increased ionization density of the light ions leads not only to a dose which increases with depth, but also to a change in the quality of the radiation. Radiation at high-LET values has an increasing relative biological efficiency (RBE: the ratio of doses for a given survival rate at different LET) up to about 200 $\mathrm{keV}$ per micron, beyond which the RBE decreases (Fig. 5). Consequently, the bio-

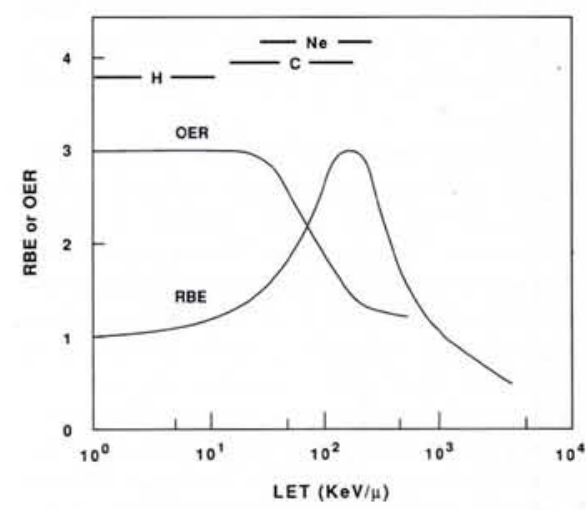

Fig. 5 - Oxygen enhancement ratio (OER: the ratio of the radiosensitivities for well oxygenated relative to poorly oxygenated cells) and the relative biological efficiency (RBE: the ratio of doses for a given survival rate at different $L E T$ ) versus linear energy energy transfer (LET). The range of LET available with $\mathrm{H}, \mathrm{C}$ and $\mathrm{Ne}$ is indicated in the upper part. 
logically effective dose at a depth relative to the dose at the entrance region increases even more than the physical dose shown in Figs. 2 and 3.

These physical and biological considerations lead to the conclusion that the optimum ions for radiotherapy are in the range from helium to neon. He ions (and protons) are the most suitable when the tumour and adjacent tissues respond more effectively to low-LET radiation. For tumours displaying a superior response to high-LET, ions in the range $\mathrm{C}$ to $\mathrm{Ne}$ appear to be the best; the lower LET at entry will then help normal tissue be spared.

\section{Accelerator Technology}

The particle energies required for $20 \mathrm{~cm}$ range in tissue are given in Table 1 , together with the intensity needed to give a typical dose within about one minute. Selecting an accelerator for proton or ion therapy is not simple because the requirements lie in the overlap between large cyclotrons and small synchrotrons. Therapy has up to now usually been carried out with machines originally built for nuclear research, and adapted or used part of the time for medical treatment. However, more and more accelerators dedicated to medical use are coming on-stream.

For proton therapy at $60-90 \mathrm{MeV}$, useful for superficial and ocular cancers, several small cyclotrons are operating, and are supplied by at least two manufacturers. A new synchrotron has been installed for deeper treatments at the Loma Linda Hospital, USA, while several proton cyclotron systems are being developed [10].

lon therapy has been pioneered for over 12 years with the Bevalac at the Lawrence Berkeley Laboratory, California, USA [11]. This machine is primarily used for physics, and only about 100 patients can be treated each year. Based on the experience gained, several new machines dedicated to light ion therapy have been proposed: a large $100 \mathrm{M}$ \$US synchrotron is under construction at Chiba, Japan, ready for the start of operations in 1993, while a new synchrotron to replace the Bevalac has been proposed. In Europe, a feasibility study for a European Light Ion Medical Accelerator (EULIMA) has been sponsored by the European Community and carried out in collaboration with CERN which acted as the host. The study defined and costed $(\approx 50 \mathrm{M} \mathrm{ECU})$ a synchrotron and treatment facilities to deliver a beam of oxygen ions at about $400 \mathrm{MeV} /$ nucleon to 3000 patients each year [12].

\section{Beam delivery}

In conventional radiotherapy with $\mathrm{x}$-rays or $\gamma$-rays, the illuminated field is inevitably large and the end point of the range badly determined, for even if the $\mathrm{x}$-rays are well collimated outside the body they spread by scattering to several centimetres at depth. In contrast, proton beams, and

Fig. 6 - Three-dimensional conformal therapy. The Bragg peak is scanned laterally and in range to treat the whole volume of a tumour.
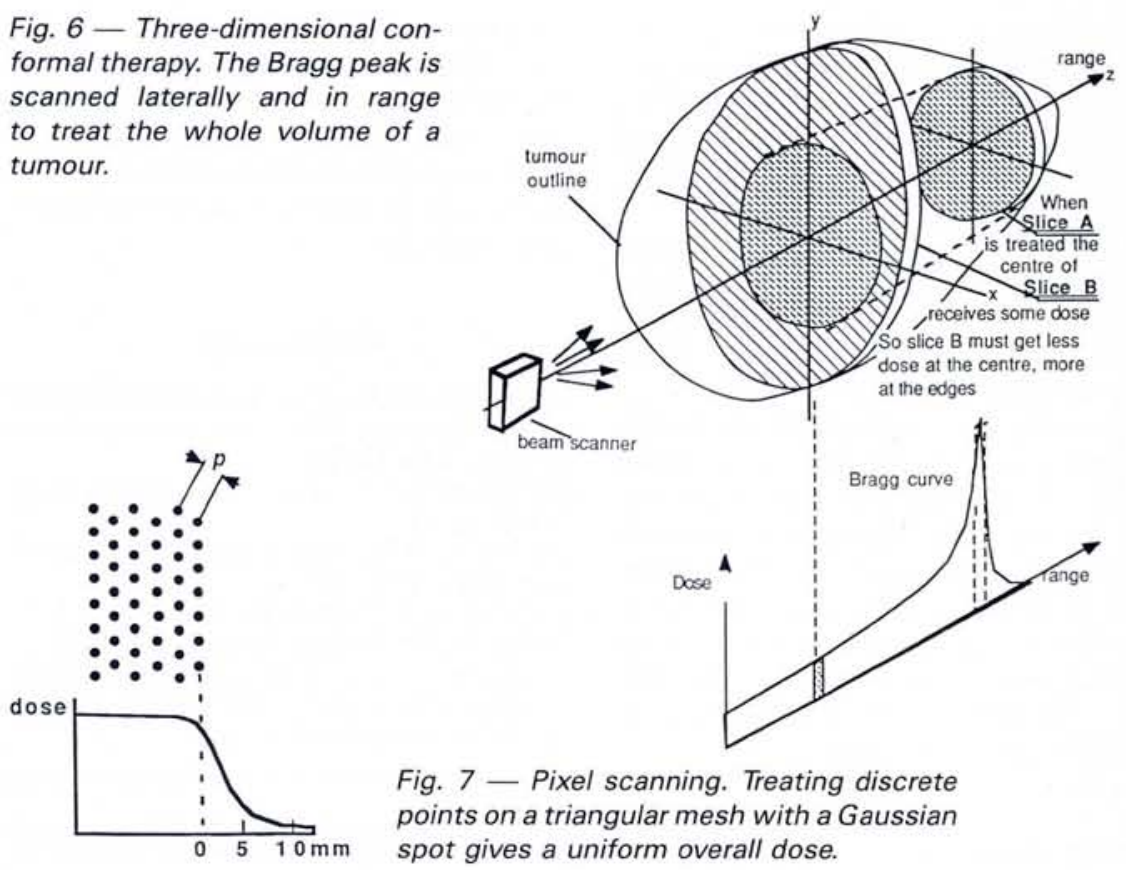

Fig. 7 - Pixel scanning. Treating discrete points on a triangular mesh with a Gaussian spot gives a uniform overall dose.

above all ion beams, can be exactly localized to a few millimetres in the lateral direction and to about $5 \mathrm{~mm}$ in range (see Fig. 1). Until now, the treatment area has been defined by collimators, but in principle a tumour can be treated more precisely by scanning in three dimensions over a designated volume of arbitrary shape. The beam delivery system must accomplish this reliably and safely at reasonable cost so the requirements are: lateral $(x-y)$ deflection by a fast magnet; variable range; variable exposure time to achieve a uniform biologically effective dose, as illustrated in Fig. 6; position sensitive monitors; and a rapid shut-down in the event of a malfunction. A corollary is that the diagnostician must determine where the tumour is with comparable precision.

Fig. 6 also shows a typical treatment plan where the Bragg curve is placed successively at various depths by adjusting the range. Each depth slice is scanned in the $x-y$ plane over the tumour cross-section, noting that when a distal slice ( $A$ in the figure) is treated, the centre of central slice (B) will receive some dose. This must be compensated for when slice $B$ is treated by giving more dose at the edges than at the centre. Therefore, each slice generally requires a carefully computed nonuniform dose, and scanning across the $x-y$

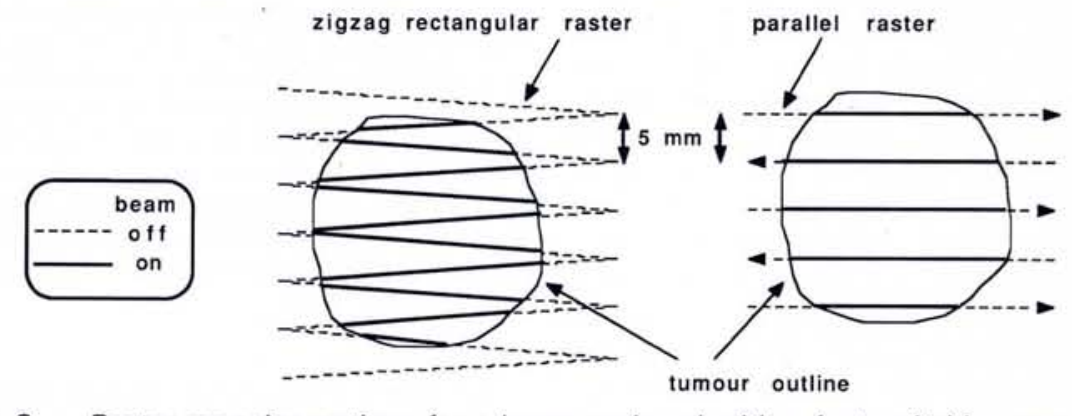

Fig. 8 - Raster scanning options for a beam equipped with a fast switching magnet.

plane may be either continuous (a socalled raster scan) or intermittent (a pixel or spot scan) [10, 13].

\section{Pixel scanning}

In pixel scanning, the target plane is treated at a triangular mesh of points (Fig. 7) with spacing $p$ : the beam spot has a Gaussian intensity with a standard deviation $s$. If $s \geq 0.5 p$ the dose is uniform to $1.2 \%$ : the edge definition depends on $s$ and is shown in Fig. 6 for $s=2.5 \mathrm{~mm}$, $p=5 \mathrm{~mm}$. These characteristics are sufficient to delineate a cross-section of arbitrary shape by choosing which pixels to treat. The procedure would then be as follows: beam off - move spot to pixel beam on until the desired dose is reached - repeat for the next pixel.

Continuous scanning strategies (Fig. 8) offer an alternative. The beam must be equipped with a fast switching magnet which dumps unwanted beam onto an absorber. With appropriate servo-control such a switch can be used, not only to turn the beam on and off, but also to regulate its intensity. One can then scan the tumour in a series of straight lines at uniform speed, adjusting the beam intensity appropriately at each point: when the spot falls outside the tumour, the beam would be turned off. Geometric considera-

Europhys. News 23 (1992) 
tions indicate that a parallel to-and-fro raster is more efficient than a zig-zag one for a field uniform to $2.8 \%$ (with a $2.5 \mathrm{~mm}$ spot size) where the raster spacing must be $5 \mathrm{~mm}$ vertically between turning points.

With a parallel to-and-fro raster, typical rise times required in the scanning magnets (specified from zero to the full deflection of $150 \mathrm{~mm}$ ) are $t$ (horizontal) $=45 \mathrm{~ms}$ and $t$ (vertical) $=300 \mathrm{~ms}$.

The peak power required for the driving electronics is $2 \mathrm{~J} / t$, where $J$ is the stored energy of the magnetic field in joules. With careful beam design the magnetic energies can be kept low, say $75 \mathrm{~J}$ for the horizontal deflector and $300 \mathrm{~J}$ for the vertical, so the peak powers $P$ for the driving electronics would be modest: $P$ (horizontal, for 75 joules) $=3.3 \mathrm{KW} ; P$ (vertical, for 300 joules) $=2.0 \mathrm{KW}$.

\section{Scanning in range}

The depth required in tissue is typically 5 to $20 \mathrm{~cm}$, shallower tumours being treated with low energy proton machines. The corresponding particle energies are 80 $180 \mathrm{MeV} /$ nucleon for protons and alpha particles, $150-330 \mathrm{MeV} /$ nucleon for carbon and 170-400 MeV/nucleon for oxygen. There is no problem in varying the extraction energy using a synchrotron. A cyclotron, however, has a fixed extraction energy so here it will be necessary to reduce the energy by passing the beam through a slab of matter (a degrader) of variable thickness. Unfortunately, the degrader has several undesirable effects which must be analyzed, including:

- increase of the beam phase space by multiple scattering;

- increase of the momentum spread by energy straggling;

- fragmentation of incoming particles, giving lighter ions of roughly the same velocity with a longer range in the patient.

The increase in phase space in a degrader depends on its thickness as well as the scattering angle [14], so the ideal material should have high density and low $Z$. The best compromise is diamond (not readily available in the required thickness); the next best are boron carbide and beryllium. For optimum results, the beam must be focussed onto the centre of the degrader at an optimum convergence angle.

With proton treatment, there is no problem of fragmentation, and the degrader can be placed just in front of the patient, so the beam line can be at fixed energy. With other ions, fragmentation inside the body is inevitable. How vital is it to eliminate fragments produced in an external degrader? This question is still debated. If necessary, the degrader could be placed far from the patient, so that most of the fragments will be lost in the subsequent bending magnets; but then the beam design becomes more complicated.

\section{Conclusions}

Interest in cancer treatment by protons and light ions is increasing rapidly. In the next decade, we can expect to see several more proton accelerators installed in hospitals, and at least one centre for treatment with ions.

\section{REFERENCES}

[1] Wambersie A., Proc. EULIMA Workshop on the Potential Value of Light lon Beam Therapy, Nice (1988).

[2] Mandrillon P. et al., Proc. EPAC, Nice (1990) 1473.

[3] Koehler A.M. and Preston W.M., Radiology 104 (1972) 191.

[4] Castro J.P., Chen G.T.Y. and Blakely E.A. (1985) Radiation Res. 104 (1985) 5263.

[5] Kouris K., Spyron N. and Jackson D.F., Imaging with lonizing Radiations (Surrey University Press, UK) 1982.
[6] Raju M.R., Heavy Particle Radiotherapy (Academic Press) 1980.

[7] Larsson B., Ref. [1], p. 139.

[8] Tubiana M., Dutreix J. and Wambersie A., Radiobiologie (1986); Wambersie A., Barendsen G.W. and Breteau N., Eur. J. Rad. Oncol. 5 (1984) 248.

[9] Curtis H.J., Current Topics in Radiation Res. 3 (1987) 139.

[10] Jongen Y. et al., Ref. [2], p. 274; Blosser $\mathrm{H}$. et al., Michigan State University $\mathrm{Cy}$ clotron Laboratory Report, MSUCL-706a

(1991); Pedroni E. et al., Ref. [2], p. 277.

[11] Castro J.R., Third Workshop on Heavy Charged Particles in Biology and Medicine,

GSI Darmstadt Report 155 N0171-4546 (1987) KOI-5.

[12] Mandrillon P. et al., Ref. [2], p. 1790; Lefèvre P. et al., CERN/PS Internal Report (1991).

[13] Kawashi K. et al., Acta Radio. S364 (1983) 81.

[14] Carli C. and Farley F.J.M., Ref. [2], p. 1796.

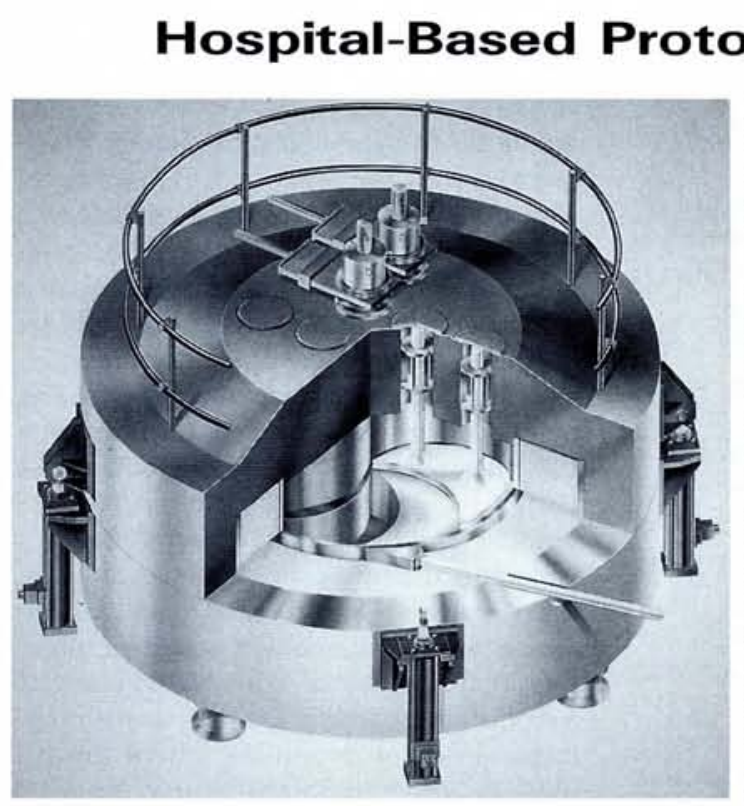

It is generally agreed that among the different kinds of radiation suitable for medical radiotherapy, high energy proton beams exhibit the best ballistic specificity for the selective destruction of cancer cells. Development has been hindered, however, by the size, cost and complexity of the equipment. To overcome these obstacles, a handful of companies are directing research at designing and supplying not only an accelerator, but also turn-key, easily operated, proton therapy facilities for hospitals.

Proton therapy has up to now been carried out at essentially physics-based installations in Zurich, Uppsala, Paris, Nice, Harvard and Berkeley in the USA and Tsukuba and Chiba in Japan. The Commission of the European Community optimistically estimates that some 10 hospital-based facilities are required in Europe over the next 10 years. Longer term interest will be determined by the demonstrated efficacy level of hospital-based proton therapy and whether doctors become convinced scanning systems are foolproof.

\section{IBA Design}

Ion Beam Applications (IBA) is among those companies with the most advanced traction), deep-valley design using $190 \mathrm{~kW}$ in conventional coils. The upper half of the split cyclotron can be quickly raised one metre using hydraulic jacks to allow unrestricted access. This feature, combined with a short pump-down time (30 min), minimizes downtime. To increase the reliability of the system, the number of components is reduced to a minimum.

An unique extraction scheme deflects particles in the hill region so that they pass through a specific "window" at the level of the closed pole edges beyond which the beam encounters a magnetic field gradient at a level below $2.2 \mathrm{~T}$, thus avoiding the use of an expensive electromagnetic tunnel.

The cyclotron would feed 2-4 isocentric gantries of reduced size and cost which allow the the proton beam to scan a patient using both conventional scanning techniques and a novel approach that links the axial sweep magnet field to a dose integrator, guaranteeing uniform dose irrespective of small intensity fluctuations. For conventional step ("voxal") scanning, the new gantry design makes it possible to achieve an infinite source-to-patient distance with a gantry of less than $3 \mathrm{~m}$ in radius. 